Research Paper

\title{
Exosomes Secreted from Human-Induced Pluripotent Stem Cell-Derived Mesenchymal Stem Cells Prevent Osteonecrosis of the Femoral Head by Promoting Angiogenesis
}

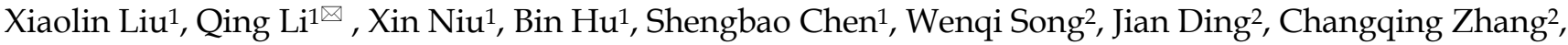 \\ Yang Wang1 ${ }^{\boxplus}$ \\ 1. Institute of Microsurgery on Extremities, Shanghai Sixth People's Hospital Affiliated to Shanghai Jiaotong University, 600 Yishan Road, Shanghai 200233, China. \\ 2. Department of Orthopedic Surgery, Shanghai Sixth People's Hospital Affiliated to Shanghai Jiaotong University, 600 Yishan Road, Shanghai 200233 , China. \\ $\triangle$ Corresponding authors: Shanghai Sixth People's Hospital Affiliated to Shanghai Jiaotong University, 600 Yishan Road, Shanghai 200233, China. Tel: \\ +86-21-64369181. Fax: +86-21-64701361. Email addresses: wangyang63@sjtu.edu.cn (Y.Wang), liqing_236@aliyun.com (Q.Li). \\ (C) Ivyspring International Publisher. This is an open access article distributed under the terms of the Creative Commons Attribution (CC BY-NC) license \\ (https://creativecommons.org/licenses/by-nc/4.0/). See http://ivyspring.com/terms for full terms and conditions.
}

Received: 2016.07.23; Accepted: 2016.12.01; Published: 2017.02.06

\begin{abstract}
Background: Local ischemia is the main pathological performance in osteonecrosis of the femoral head (ONFH). There is currently no effective therapy to promote angiogenesis in the femoral head. Recent studies revealed that exosomes secreted by induced pluripotent stem cell-derived mesenchymal stem cells (iPS-MSC-Exos) have great therapeutic potential in ischemic tissues, but whether they could promote angiogenesis in ONFH has not been reported, and little is known regarding the underlying mechanism.

Methods: iPS-MSC-Exos were intravenously injected to a steroid-induced rat osteonecrosis model. Samples of the femoral head were obtained 3 weeks after all the injections. The effects were assessed by measuring local angiogenesis and bone loss through histological and immunohistochemical (IHC) staining, micro-CT and three-dimensional microangiography. The effects of exosomes on endothelial cells were studied through evaluations of proliferation, migration and tube-forming analyses. The expression levels of angiogenic related PI3K/Akt signaling pathway of endothelial cells were evaluated following stimulation of iPS-MSC-Exos. The promoting effects of exosomes were re-evaluated following blockade of PI3K/Akt.

Results: The in vivo study revealed that administration of iPS-MSC-Exos significantly prevented bone loss, and increased microvessel density in the femoral head compared with control group. We found that iPS-MSC-Exos significantly enhanced the proliferation, migration and tube-forming capacities of endothelial cells in vitro. iPS-MSC-Exos could activate PI3K/Akt signaling pathway in endothelial cells. Moreover, the promoting effects of iPS-MSC-Exos were abolished after blockade of PI3K/Akt on endothelial cells.

Conclusions: Our findings suggest that transplantation of iPS-MSC-Exos exerts a preventative effect on ONFH by promoting local angiogenesis and preventing bone loss. The promoting effect might be attributed to activation of the PI3K/Akt signaling pathway on endothelial cells. The data provide the first evidence for the potential of iPS-MSC-Exos in treating ONFH.
\end{abstract}

Key words: induced pluripotent stem cells (iPSCs), Exosomes, Osteonecrosis, Femoral head, Angiogenesis.

\section{Introduction}

Osteonecrosis of the femoral head (ONFH) mainly affects young and middle aged people. In America, the prevalence of ONFH was 10,000 to
20,000 new cases annually [1]. The etiology of ONFH is complicated, but all the risk factors are related to ischemia of femoral head. For example, trauma can 
lead to vascular interruption, alcohol or corticosteroids overuse can result in fat emboli, and intravascular coagulation can lead to thrombotic occlusion. These ischemic conditions finally result in osteocyte necrosis and loss of structural integrity of the femoral head [2, 3]. Current therapies are composed of either conservative or surgical treatment, such as weight bearing avoidance, anticoagulants or antiosteoporosis drugs, and core decompression. These treatments only attempt to relieve symptom, whereas they can not prevent the progress of ischemia and subsequent femoral head collapse. Patients in the late stage usually have to undergo total hip replacement, which may lead to many associated problems, such as infection, osteolysis, dislocation and joint revision [4]. Therefore it would be of great importance to find an effective way of promoting angiogenesis in the femoral head.

Stem cells are known to have the ability to promote angiogenesis. Transplantation of stem cells can enhance the growth and invasion of vessels from surrounding tissues by promoting tube formation by endothelial cells [5]. Grunewald et al. [6] found that MSCs could recruit pericytes and smooth muscle cells to promote maturation of newly-formed blood vessels. Recent studies also identified angiogenic effects of stem cell transplantation in osteonecrosis of the femoral head. Yan et al. [7] found that transplanting mesenchymal stem cells into the femoral head contributed to acceleration of the repair process in an ONFH animal model. Tabatabaee et al. [8] found that transplantation of autologous bone marrow stem cells by injection could improve the clinical outcome in patients with early-stage ONFH. However, direct transplantation of stem cells still retains several limitations. For example, transplanted stem cells exhibit a low survival rate in ischemic tissue [9]. Other risks such as cell dedifferentiation, immune rejection, genetic modification and tumor formation also limit the clinical use of directly-transplanted stem cells for ONFH.

Recent studies showed that the therapeutic effect of stem cell transplantation was mainly due to their secretory behavior [1, 10]. Among these secreted biochemicals, exosomes seem to play an important role in tissue repair. Exosomes are nano-sized liposomes with a diameter of 40 to $100 \mathrm{~nm}$. They are assembled by invagination of the endosomal membrane in multivesicular bodies (MVBs), then released into extracellular space through fusion with the plasma membrane [11-13]. These extracellular vesicles (EVs) are involved in transportation of functional biochemicals, such as cytokines, RNAs and proteins [14]. The contents of exosome are protected by a liposomal membrane from degradation or destruction. Specific surface ligands of an exosome can bind to a target cell, delivering messages or biochemicals into the target cell to stimulate a particular biological function $[15,16]$. Transplantation of exosomes has been confirmed to exert similar therapeutic effects to direct stem cell transplantation in tissue repair $[17,18]$. Moreover, such an alternative strategy avoids all the issues caused by direct stem cell transplantation. Transplantation of exosomes secreted by stem cells might therefore be a promising treatment option for tissue repair.

Our previous studies found that transplantation of exosomes secreted by human-induced pluripotent stem cell-derived mesenchymal stem cells (hiPS-MSC-Exos) could promote angiogenesis of ischemic tissue in limb ischemia and skin defects [17, 18]. These satisfactory results suggest that transplantation of hiPS-MSC-Exos might be useful for other ischemic diseases including ONFH. Despite the promising effects of hiPS-MSC-Exos in ischemic diseases, the potential mechanism which regulates angiogenesis remains unclear. Several studies have demonstrated the potential relationship of the PI3K/Akt pathway and stem cells in angiogenesis [5]. Piao et al. [19] found that transplanting MSCs with increased expression levels of pAkt could increase angiogenesis in the ischemic limb of rats. Culture medium from MSCs has been reported to increase the level of angiogenesis by increasing the level of pAkt, an effect which could be inhibited by a PI3K/Akt inhibitor [20]. These results suggest that stem cells can increase the angiogenic ability of endothelial cells via the PI3K/Akt pathway. Since exosomes are important functional products of host cells, the angiogenic effect of exosomes secreted by stem cells might also be associated with the PI3K/Akt pathway. Based on these studies, we hypothesize that transplantation of hiPS-MSC-Exos might prevent the progression of $\mathrm{ONFH}$, and the PI3K/Akt pathway might be involved in the angiogenic effects triggered by hiPS-MSC-Exos.

\section{Methods}

\section{Preparation of hiPS-MSCs and hiPS-MSC-Exos}

\subsection{Generation of MSCs from human iPSCs}

Three human iPSC (hiPSC) lines were adopted in the generation of MSCs. The first (iPS-S-01) was provided by the Institute of Biochemistry and Cell Biology of the Chinese Academy of Sciences in agreement with Dr Liao and Prof Xiao [21]. The other two cell lines (iPSCs-C1P33 and iPSCs-PCKDSF001C1) were provided by the South China Institute for Stem Cell Biology and Regenerative Medicine Group of the Chinese 
Academy of Sciences in agreement with Prof Pei [22]. The generation of iPSC-derived MSCs was induced as previously described [17]. The iPSCs were expanded on human ESC-Qualified BD Matrigel (BD Biosciences, Franklin Lakes, NJ, USA) in mTESR1 (StemCell Technologies, Vancouver, BC, Canada) [23]. Then mTESR1 was replaced with MSC medium and the cells were cultured for 14 days. The MSC medium was composed of Dulbecco's Modified Eagle Medium (DMEM)-low glucose (Corning, Tewksbury, MA, USA) supplemented with $10 \%$ fetal bovine serum (FBS) and $2 \mathrm{mM}$ L-Glutamine (Gibco, Grand Island, NY, USA) [24]. These cells were then trypsinized (0.25\% trypsin/1 mM EDTA; Gibco) and reseeded in $0.1 \%$ gelatin-coated cell culture flasks (Corning) in MSC medium. After cultured to passage 4, the cells began to show a homogeneous fibroblastic morphology. Evaluation of the MSCs' phenotypic characteristics and differentiation potential were performed to verify the newly induced hiPS-MSCs.

\subsection{Flow cytometric analysis}

Surface antigens of hiPS-MSCs were analyzed using flow cytometry. The monoclonal antibodies used (BD Biosciences) were CD29-PE, CD34-APC, CD44-FITC, CD45-FITC, CD73-PE, CD90-PE, CD133-PE and CD146-PE. The cells were incubated with $1 \%$ bovine serum albumin (BSA; Gibco) for 30 minutes to block nonspecific antigens. Following two washes in PBS, the cells were resuspended and analyzed using the Guava easyCyte ${ }^{\mathrm{TM}}$ system (Millipore, Billerica, MA, USA). Nonspecific fluorescence was determined with isotype-matched mouse monoclonal antibodies (BD Biosciences).

\subsection{Isolation and identification of hiPS-MSC-Exos}

\subsubsection{Isolation of hiPS-MSC-Exos}

Exosomes were isolated from hiPS-MSC supernatants as previously described $[25,26]$. After reaching $80 \%$ confluence, the cells were rinsed three times with PBS, then cultured in a chemically-defined medium (MesenGro hMSC medium; StemRD, San Francisco, CA, USA) for 48 hours. The conditioned medium $(\mathrm{CM})$ was collected, centrifuged at $300 \times \mathrm{g}$ for $10 \mathrm{~min}, 2000 \times \mathrm{g}$ for $10 \mathrm{~min}$ at $4^{\circ} \mathrm{C}$, then filtered through a $0.22-\mu \mathrm{m}$ Sterilize Steritop ${ }^{\mathrm{TM}}$ filter (Millipore) to remove cellular debris. The supernatants were then ultracentrifuged at $100,000 \times g$ for 2 hours to collect exosomes. Exosomes in the pellet were resuspended in PBS, transferred to the upper compartment of an Amicon Ultra-15 Centrifugal Filter Unit (Millipore) and centrifuged at $4000 \times g$ at $4^{\circ} \mathrm{C}$ until the volume in the upper compartment was reduced to approximately $200 \mu \mathrm{L}$. An equal volume of control medium was obtained from fresh MesenGro hMSC medium in the same way as the collection of exosomes.

\subsubsection{Identification of hiPS-MSC-Exos}

Transmission electron microscopy (TEM) was used to examine the morphology of hiPS-MSC-Exos. Briefly, hiPS-MSC-Exos were fixed in 3\% glutaraldehyde for 2 hours, washed twice with PBS, then negatively stained with $2 \%$ uranyl acetate for 30 seconds and applied to a continuous carbon grid. The morphology of hiPS-MSC-Exos was visualized with a Hitachi H-7650 transmission electron microscope (Hitachi, Tokyo, Japan), and the images were captured using a digital camera (Olympus, Tokyo, Japan).

Western blot analysis was performed to identify surface markers of hiPS-MSC-Exos, including CD9, CD63, and CD81 [27]. The samples were lysed in protein extraction reagent (Pierce, Thermo Fisher Scientific, Waltham, MA, USA) supplemented with protease inhibitor. Total protein contents were determined with the Pierce BCA Protein Assay Kit (Pierce). The samples were loaded onto $10 \%$ SDS polyacrylamide gels (SDS-PAGE) and transferred to a polyvinylidene difluoride membrane (PVDF: Millipore). The membrane was blocked with $1 \%$ BSA (Gibco) followed by incubation with the primary antibodies rabbit polyclonal anti-CD9, anti-CD63 and anti-CD81 (Abcam, Cambridge, UK). The proteins were detected using enhanced chemiluminescence (Thermo Fisher) and the images were captured using an Image Quant LAS 4000 mini bio-molecular imager (GE Healthcare, Little Chalfont, UK). Nanoparticle analysis was performed to identify size and concentration of hiPS-MSC-Exos with a qNano platform (iZON, Cambridge, MA, USA). Data processing was performed using Control Suite software v2.2 (iZON).

\section{Therapeutic effect of hiPS-MSC-Exos in vivo}

\subsection{Administration of exosomes to a rat steroids-induced ONFH model}

All in vivo experimental procedures were approved by the Animal Research Committees of Shanghai Sixth People's Hospital. Forty adult male SD rats weighting 300-320g were used in the study. The rats were randomly divided into four groups: MP group (treated with steroids to induce ONFH, $\mathrm{n}=10$ ), $\mathrm{MP}+$ Exosomes group (treated with steroids and different concentrations of hiPS-MSC-Exos, $n=10$ ), Control group (treated with an equal volume of control medium, $\mathrm{n}=10$ ), and normal control group ( $\mathrm{n}$ $=10)$. The ONFH model was created by treatment with steroids using a modified method based on previous reports [28, 29]. Briefly, methylprednisolone 
acetate (MP, Pfizer Manufacturing, Puurs, Belgium) $(40 \mathrm{mg} / \mathrm{kg})$ was injected intramuscularly for three times per week for 3 weeks to induce ONFH. In the $\mathrm{MP}+$ Exosomes group, tail vein injection was performed with $100 \mu \mathrm{L}$ of hiPS-MSC-Exos $(1 \times$ $10^{10} / \mathrm{mL}$ or $1 \times 10^{11} / \mathrm{mL}$ ) before each MP injection. In the Control group, the rats received tail vein injection with $100 \mu \mathrm{L}$ of control medium. After completing the course of injections, the rats were fed a standard diet and allowed free activity for another 3 weeks. Then the femoral heads of all the rats were collected to evaluate osteonecrosis and the treatment effects of exosomes by micro CT, micro-CT-based micro-angiography, histological and immunohistochemical examination.

\subsection{Micro-CT}

Micro-CT (Skyscan, 1076 scanner, Kontich, Belgium) images were obtained to evaluate trabecular bone structure of the femoral head. The dissected, formalin-fixed samples were scanned with the femur axis perpendicular to the plane at $18 \mathrm{~mm}$ resolution. The 3D representation of the regenerated bone was reconstructed with NRecon software (Version 1.5.1.4, Skyscan). Based on the CT images, a region of interest (ROI) was selected for comparison among groups, and values of bone volume/total volume (BV/TV), bone surface area/bone volume, trabecular thickness (Tb.Th) and trabecular number (Tb.N) were compared.

\subsection{Micro-CT-based micro-angiography (CTA)}

Vascularization in the femoral head was evaluated via CTA analysis as previously described $[30,31]$. Briefly, the thoracic and abdominal cavities were cut open after the rats were successfully anaesthetized. The proximal branch of the ascending aorta was ligated, then a hypodermic needle with disposable infusion device was inserted into the left ventricle. Heparinized saline was used to flush the vasculature until all the blood was washed out, and $4 \%$ paraformaldehyde solution was used to fix the tissues and blood vessels. The vasculature was then injected with Microfil according to the manufacturer's protocol (Microfil MV-122, Flow Tech, Carver, MA, USA). The rats were stored overnight at $4{ }^{\circ} \mathrm{C}$, then both femoral heads were harvested, fixed in $4 \%$ paraformaldehyde, then transferred into 10\% EDTA for decalcification. Finally, the samples were observed by micro-CT scanning with a Skyscan, 1076 scanner, and the data were analyzed using NRecon software (Version 1.5.1.4, Skyscan).

\subsection{Histological and immunohistochemical (IHC) analysis}

For histological analysis, the samples were fixed in $4 \%$ formaldehyde, decalcified in $10 \%$ EDTA solution and then embedded in paraffin. The embedded samples were cut into $5 \mu \mathrm{m}$ sections with a microtome (Leica Microsystems, Wetzlar, Germany). Sections were stained with hematoxylin and eosin (HE) and visualized under a light microscope (Leica Microsystems). The extent and severity of osteonecrosis was evaluated according to changes in the trabecular bone by two researchers who were blinded to the identity of the samples. Local osteonecrosis was determined according to the presence of empty lacunae or pyknotic nuclei of osteocytes within the trabecular bone, as well as the thickness and density of the trabecular bone. Under $200 \times$ magnification, 10 fields were randomly chosen and 20 bone lacunae were counted in each field. The number of empty bone lacunae and the ratio of empty bone lacunae versus total bone lacunae were compared within each group.

For IHC analysis, expression of VEGFR2 and CD31 were observed to evaluate angiogenesis of the femoral head. Paraffin sections were dewaxed by a routine method, incubated for 10 min with $\mathrm{H}_{2} \mathrm{O}_{2}$ and for 30 min with 1\% BSA (Gibco) at room temperature, then incubated with primary rabbit anti-VEGFR2 and anti-CD31 antibodies (1:200; Abcam) at $4^{\circ} \mathrm{C}$ overnight. Following three washes in PBS, the sections were incubated with poly-HRP goat anti-rabbit IgG for one hour at $37^{\circ} \mathrm{C}$. Then the sections were stained with 3, 3-diaminobenzidine (Sigma-Aldrich, St. Louis, MO, USA) and counterstained with hematoxylin. The positively-stained images were visualized under a light microscope (Leica Microsystems) and the number of microvessels was calculated according to the previously described procedure [32]. Briefly, under $200 \times$ magnification, 10 optical fields were randomly chosen, and microvessels were counted, with a single endothelial cell or cluster of endothelial cells considered one countable microvessel.

\section{Administration of hiPS-MSC-Exos to endothelial cells in vitro}

\subsection{Endocytosis of exosomes by endothelial cells}

Human umbilical vascular endothelial cells (HUVECs) were harvested from human umbilical cords after obtaining informed consent from donors. The obtained cells were cultured in M200 supplemented with 2\% low serum growth supplement (M200+LSGS) (Cascade Biologics, Portland, OR, USA), then inoculated into a 48-well plate at a density of $1 \times 10^{5} / \mathrm{mL}$ per well.

hiPS-MSCs were labeled with $\mathrm{DiO}$ (green) dye (Molecular Probes; Vybrant, Carlsbad, CA, USA) according to the manufacturer's protocol. Briefly, the cells were trypsinized and resuspended in $1 \mathrm{~mL}$ of 
MGro-500 serum-free MSC medium (Gibco) containing $5 \mu \mathrm{L}$ of the cell-labeling solution, followed by incubation at $37^{\circ} \mathrm{C}$ for 15 minutes. The cell suspension was centrifuged at $300 \times g$ for 5 minutes, resuspended and washed three times with PBS, then cultured in MGro-500 serum-free MSC medium for an additional 24 hours. The cultured medium was collected to isolate DiO-labeled hiPS-MSC-Exos. The obtained exosomes were then incubated with HUVECs in the 48-well plate for 2 hours. The cultured HUVECs were then washed twice with PBS, fixed with $4 \%$ paraformaldehyde for 15 minutes, and stained with DAPI for 5 minutes at room temperature. After washing twice with PBS, the cells were visualized under a fluorescence microscope (Leica Microsystems).

\subsection{Migration and proliferation assay}

The scratched wound assay was performed to evaluate migration ability as previously described [17]. Briefly, HUVECs were seeded into 12-well plates at a density of $2 \times 10^{5}$ cells per well and grown to $100 \%$ confluence. The cells were cultured in M200+LSGS containing $1 \times 10^{10} / \mathrm{mL}$ hiPS-MSC-Exos for 8 or 12 hours. The control cells were cultured in M200+LSGS without exosomes for 8 or 12 hours. Two parallel scratches were then made in each well with a $200 \mu \mathrm{L}$ pipette tip, and the width of the scratch was measured as the baseline [33]. The width of the wound was measured by mimicking an exclusion zone assay using Image J software (National Institutes of Health, Bethesda, MD, USA), and visualized with a light microscope (Leica, Microsystems).

The proliferation ability of hiPS-MSC-Exos was evaluated using a CCK-8 kit (Dojindo, Laboratories, Kumamoto, Japan) according to the manufacturer's instructions. HUVECs were seeded into 96-well plates at a density of 5000 cells per well. The cells were cultured with $100 \mu \mathrm{L}$ of M200+LSGS containing different concentrations of hiPS-MSC-Exos $\left(0,1 \times 10^{10}\right.$, $1 \times 10^{11} / \mathrm{mL}$ ). From day 1 to day $5,10 \mu \mathrm{L}$ of CCK-8 solution was added to each well, and plates were then incubated at $37^{\circ} \mathrm{C}$ for $3 \mathrm{~h}$. The absorbance at $450 \mathrm{~nm}$ was measured using a microplate reader (Bio-Rad

Table 1. Primers used for quantitative reverse-transcriptase polymerase chain reaction.

\begin{tabular}{lll}
\hline Genes & Forward primer $\left(5^{\prime}-3^{\prime}\right)$ & Reverse primer $\left(5^{\prime}-3^{\prime}\right)$ \\
\hline h-BCL2A1 & TCAGTGGCCCACAAGAAGAG & GGAGTGTCCTTTCTGGTCAACA \\
h-SPHK1 & CTGGGCAGCACCGATAAGG & TCCGCAACCGACCACTG \\
h-MYC & TTACAACACCCGAGCAAGGA & AAATACGGCTGCACCGAGTC \\
h-THBS1 & GAGAATGCTGTCCTCGCTGT & AGCCCTCACATCGGTTGTT \\
h-PTEN & CAAGATGATGTTGAAACTATTCCAATG & CCTTTAGCTGGCAGACCACAA \\
h-RBL2 & GCATGAGCGAAAGCTACACG & TCCACTGTCCCTTGCTTACA \\
h-CDKN1A & TGGTAGGAGACAGGAGACCTCTAA & TTGGAACACAGGACTTTGCCT \\
h-GAPDH & ATCCCATCACCATCTTCC & GAGTCCTTCCACGATACCA \\
\hline
\end{tabular}

Laboratories, Hercules, CA, USA). All experiments were performed in triplicate and repeated 3 independent times.

\subsection{Capillary network formation assay}

Tube formation assay was performed to investigate endothelial cell network formation as previously described [17]. Briefly, HUVECs were seeded onto Matrigel ${ }^{\mathrm{TM}}$. (BD Biosciences) coated 24 well plates at a density of $5 \times 10^{4}$ cells per well. The cells were cultured in M200+LSGS containing different concentrations of hiPS-MSC-Exos $\left(0,1 \times 10^{10}\right.$, $1 \times 10^{11} / \mathrm{mL}$ ) at $37^{\circ} \mathrm{C}$ with $5 \% \mathrm{CO}_{2}$ for 4 or 8 hours. Images were captured at each time-point. The number of branch points and tubule lengths were quantified in 4 randomly-chosen fields.

\section{Identification of PI3K/Akt signaling pathway activation}

\subsection{Quantitative real-time PCR (qRT-PCR) and western blot analysis}

HUVECs were seeded into $25-\mathrm{cm}^{2}$ cell culture flasks and cultured in medium 200 (M200, Gibco) containing $1 \times 10^{11} / \mathrm{mL}$ hiPS-MSC-Exos or an equal volume of $\mathrm{PBS}$ at $37^{\circ} \mathrm{C}, 5 \% \mathrm{CO}_{2}$ for 24 hours. Expression levels of genes and proteins in the PI3K/Akt signaling pathway were identified by qRT-PCR and western blot.

For qRT-PCR, total RNAs was extracted using Trizol reagent (Invitrogen, Carlsbad, CA, USA), and reverse-transcribed into cDNAs by the RevertAid first-strand cDNA synthesis kit (Fermentas, Life Sciences, Burlington, ON, Canada). qRT-PCR analysis was conducted using the ABI PRISM ${ }^{\circledR 7900 H T ~ S y s t e m ~}$ (Takara Biotechnology Inc., Shiga, Japan). GAPDH was employed as the housekeeping gene for internal normalization. Primers for the amplification reaction are listed in Table 1.

For western blotting, the cells were lysed in protein extraction reagent (Pierce) supplemented with protease inhibitor. Total protein contents were determined with the Pierce BCA Protein Assay Kit (Pierce). The samples were loaded onto $10 \%$ SDS polyacrylamide gels (SDS-PAGE) and transferred to a PVDF membrane (Millipore). The membrane was blocked with 1\% BSA (Gibco) for 2 hours, then incubated with primary antibodies (Abcam) at $4^{\circ} \mathrm{C}$ overnight, followed by incubation with horseradish peroxidase (HRP)-labeled secondary antibodies (Cell Signaling Technology, Danvers, MA, USA) at $37^{\circ} \mathrm{C}$ for 1 hour. The proteins were detected using enhanced 
chemiluminescence (Thermo Fisher) and images were captured using an Image Quant LAS 4000 mini bio-molecular imager (GE Healthcare).

\subsection{PI3K/Akt signaling inhibition}

To confirm whether hiPS-MSC-Exos could activate the PI3K/ Akt signaling pathway in HUVECs, a highly selective inhibitor of PI3K (LY294002, Sigma-Aldrich) was used according to the manufacturer's protocol. The inhibitor was dissolved in dimethyl sulfoxide (DMSO) at a stock concentration of $100 \mathrm{mM}$. Cultured HUVECs were divided into three groups, comprising the inhibitor group, exosomes group and control group. In the inhibitor group, HUVECs were pre-treated with the indicated concentration $(10 \mu \mathrm{M})$ of LY294002 for 1 hour. Then the cells were cultured with exosomes at 1 $\times 10^{11} / \mathrm{mL}$. In the exosomes group, the cells were directly cultured with $1 \times 10^{11} / \mathrm{mL}$ of exosomes. In the control group, the cells were pre-treated with DMSO for 1 hour, then treated with PBS. qRT-PCR, western blotting, migration, proliferation and capillary network formation assays were performed on the treated HUVECs as described above.

\section{Statistical analysis}

All data are shown as mean \pm standard deviation (SD). Differences between groups were assessed by one-way analysis of variance (ANOVA). Statistical analyses were performed using SPSS 16.0 software
(SPSS, Inc., Chicago, IL, USA). $P$ values $<0.05$ were considered statistically significant.

\section{Results}

\section{Characterization of hiPS-MSCs and hiPS-MSC-Exos}

MSCs were successfully induced from three different hiPSC cell lines using the modified one-step induction protocol [24]. The cells displayed a homogeneous fibroblastic-like morphology (Fig 1A). Flow cytometric analysis was conducted to detect the expression of cell-specific antigens by these cells. The results revealed that the cells were highly positive for the MSC characteristic markers CD29, CD44, CD73, CD90, and CD146, and negative for the antigens CD45, CD34 and CD133 (Fig 1B). TEM, western blotting and nanoparticle analysis were used to characterize the purified particles derived from hiPS-MSCs. TEM images showed that exosomes exhibited spheroidal morphology and ranged in size from 30 to 100nm (Fig 1C). The results of western blotting confirmed the expression of CD9, CD63, and CD81 in exosomes, which are surface markers exceptionally enriched in exosomes (Fig 1D). Nanoparticle analysis revealed that the size of exosomes mainly ranged from 50 to $100 \mathrm{~nm}$, with a mean concentration of $3.4 \times 10^{12}$ particles $/ \mathrm{mL}$ (Fig 1E).

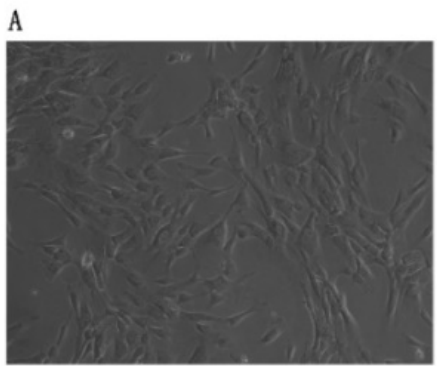

C

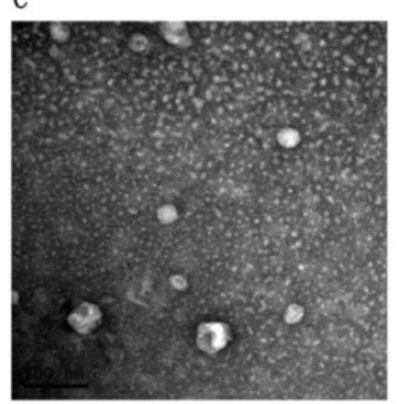

B

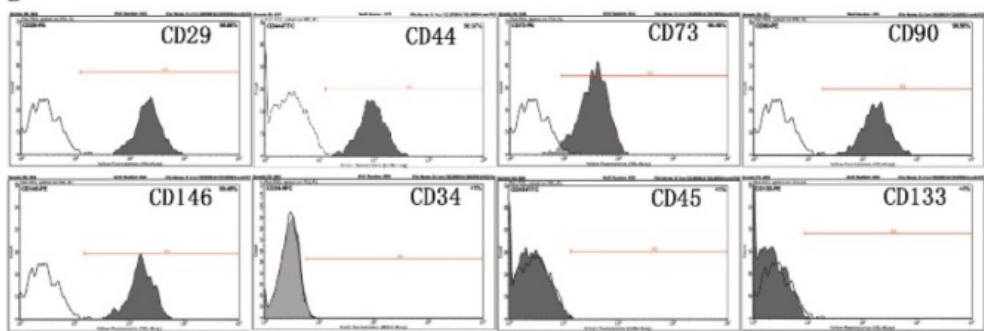

D

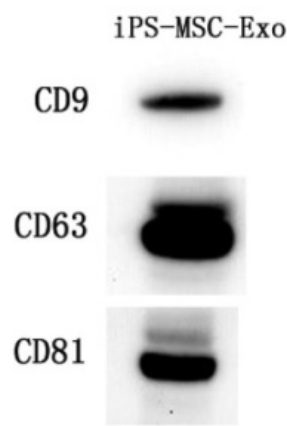

$\mathrm{E}$

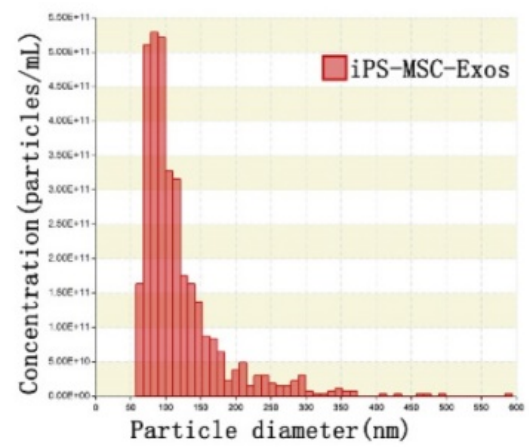

Figure 1. Characterization of hiPS-MSCs and hiPS-MSC-Exos. (A) The fibroblast-like morphology of hiPS-MSCs shown in light microscopy images. (B) Surface markers of hiPS-MSCs analyzed by flow cytometry. (C) The morphology of hiPS-MSC-Exos shown by transmission electron microscopy. (D) Expression levels of CD9, CD63 and CD81 incorporation into hiPS-MSC-Exos shown by western blotting. (E) Identification of size and concentration of hiPS-MSC-Exos by nanoparticle analysis. 


\section{Preventative effect of hiPS-MSC-Exos in a rat ONFH model}

The results in vivo analysis revealed that hiPS-MSC-Exos significantly prevented bone loss, and increased angiogenesis of the femoral head in the rat ONFH model.

\section{1. hiPS-MSC-Exos prevent bone loss in the femoral head in ONFH}

Trabecular bone structure of the femoral head was observed by HE staining and micro-CT. In the MP group, HE staining revealed that trabecular bone became sparse and thin. Empty lacunae and pyknosis of osteocytes were observed in the cavities of the trabecular bone, while necrosis of hematopoietic cells and adipose cells was found in the bone marrow. In the MP+Exo group, only slight or no osteonecrosis of the trabecular bone was observed in the femoral head, along with fewer empty lacunae and necrotic medullary hematopoietic cells and adipose cells (Fig. 2A). hiPS-MSC-Exos significantly reduced the incidence of ONFH. None of the rats developed $\mathrm{ONFH}$ in the MP+Exo group, while 8 out of 10 rats developed ONFH in the MP group $(P<0.05)$. The rate of empty lacunae in the MP+Exo group was $14.5 \pm$

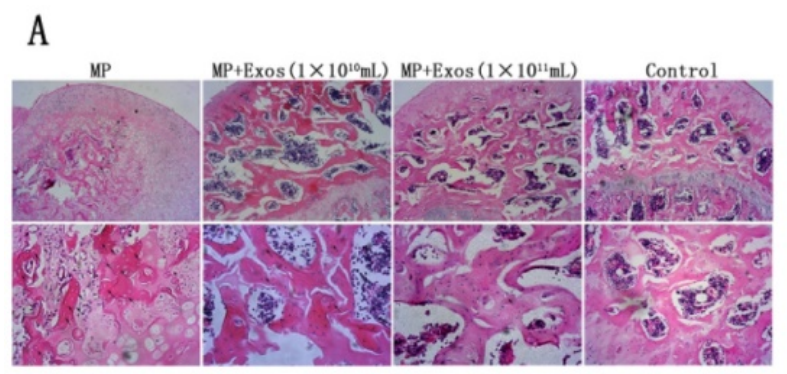

B

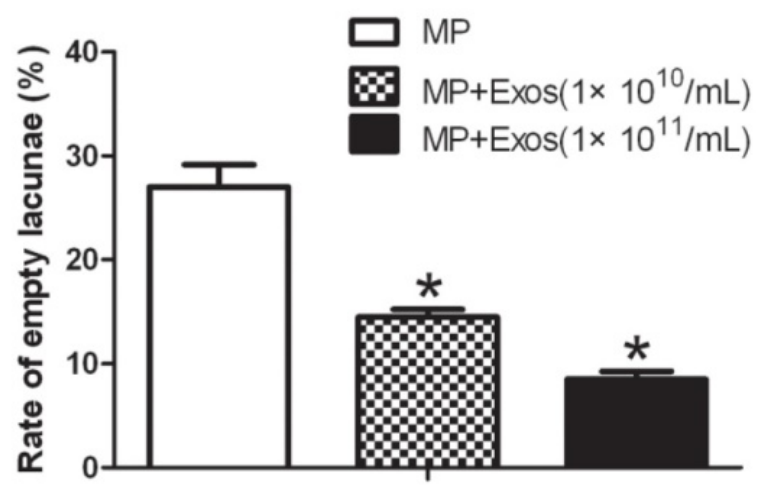

$2.4 \%$ (Fig. 2B), which was significantly lower than that in the MP group at $31 \pm 4.3 \%(P<0.05)$.

For micro-CT analysis, a region of interest(ROI) in the femoral head was selected for observation and statistical analysis. In the MP group, the trabecular bone in the femoral head was sparse and relatively thin, and in some areas had completely vanished. In the MP+Exo group the trabecular bone showed a better structural integrity (Fig 2C). Quantitative analysis showed that BV/TV, bone surface area/bone volume, Tb.Th and Tb.N were all significantly higher in the MP+Exo group compared to that of the MP group $(P<0.05$ for all variables, Fig. 2D).

\section{2. hiPS-MSC-Exos enhance angiogenesis in the femoral head in ONFH}

Angiogenesis of the femoral head was evaluated via 3-D microangiography and IHC staining. For 3-D microangiography, the images revealed significantly more vascular branches in the femoral head in the Exo group compared with the control group (Fig. 3A). The values of vessel volume, percentage of vessel volume, vessel surface and vessel thickness were all significantly increased in the Exo group compared with the control group (Fig. 3B).
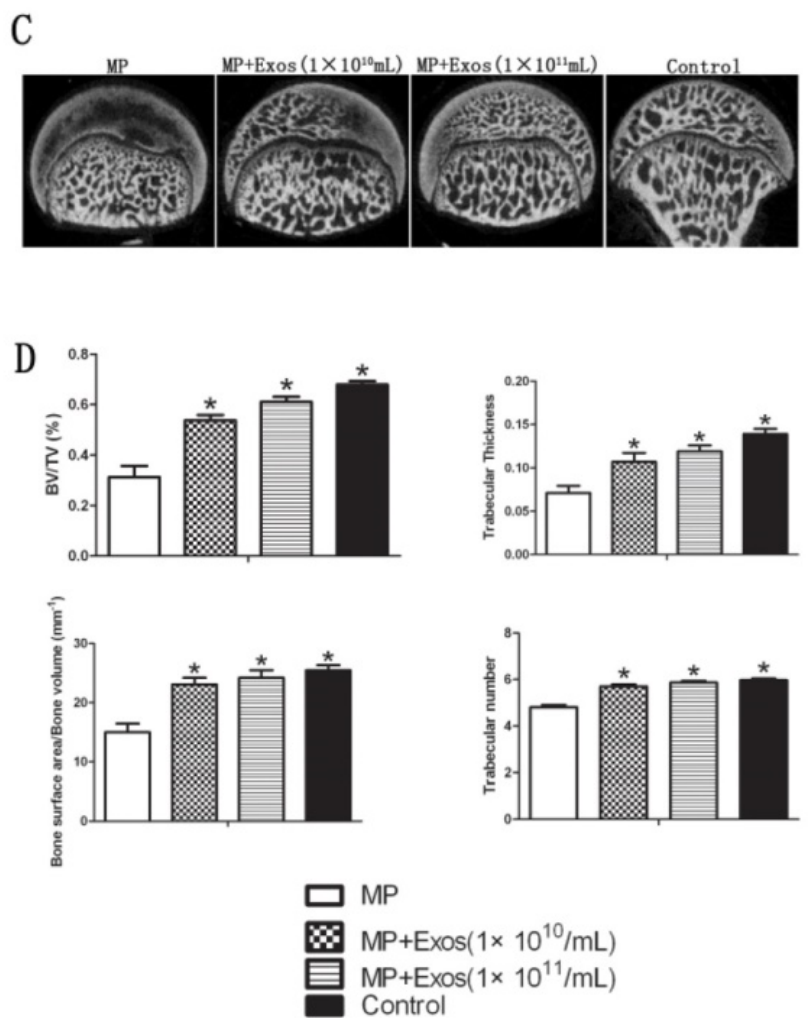

Figure 2. Assessment of trabecular bone structure in the femoral head. (A) H\&E staining analysis. More bone trabecular tissue and fewer empty lacunae were observed in the MP+Exo group compared with the MP group. Arrowheads indicate empty lacunae. (B) Quantitative analysis of $\mathrm{H} \& \mathrm{E}$ staining. The number of empty lacunae in the $M P+$ Exo group was significantly less than that in the MP group $(P<0.05)$. (C) Micro-CT analysis. The subchondral trabeculae appeared much more intact and regular in the MP+Exo group than in the MP group. (D) Quantitative analysis by mirco-CT. The values of BV/TV, bone surface area/bone volume, Tb.Th and Tb.N in the MP+Exo group were significantly higher than in the MP group $(P<0.05)$. 
A

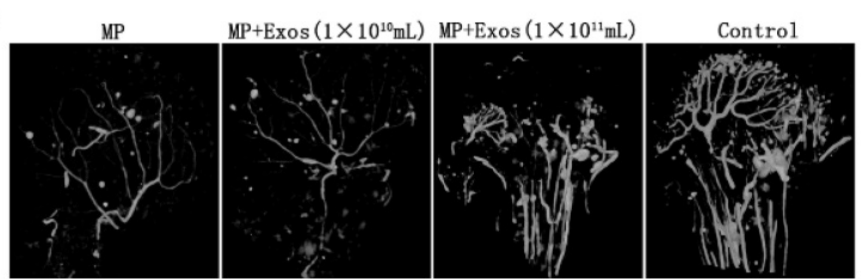

B
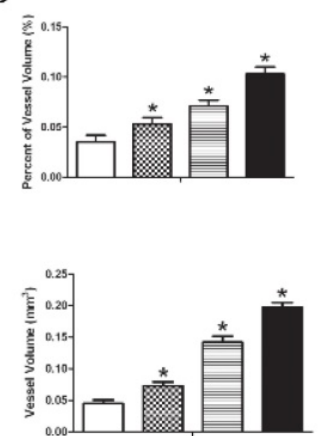
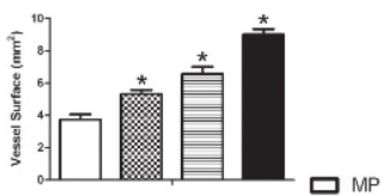

$\mathrm{OMP}$

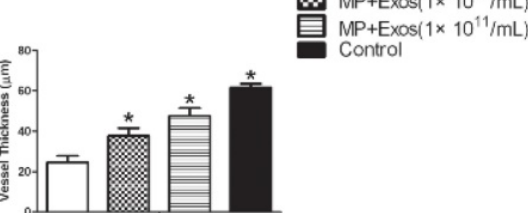

C

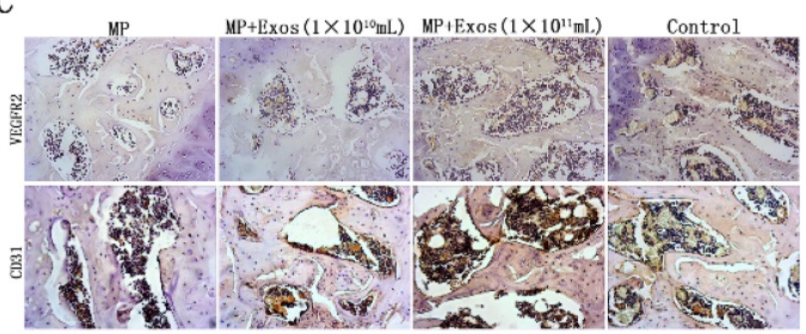

D

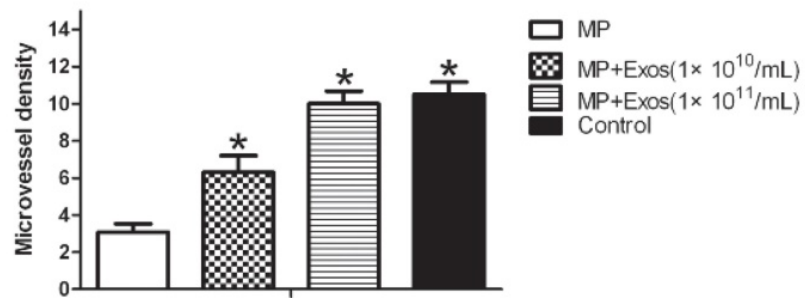

Figure 3. Assessment of angiogenesis in the femoral head. (A) 3-D microangiography analysis. Greater angiogenesis was observed in the MP+Exo group compared with the MP group. (B) Quantitative analysis of microangiography. Both vessel number and vessel volume in the MP+Exo group were significantly higher than in the MP group. (C) Immunohistochemical analysis. The expression of both VEGFR2 and CD31 was significantly enhanced in the MP+Exo group compared with the MP group.

The results of IHC showed increased expression of VEGFR2 and CD31 in the femoral head in the $\mathrm{MP}+$ Exo group compared with the MP group (Fig. 3C). Microvessel density (MVD) was calculated following IHC staining by counting single endothelial cells or clusters of endothelial cells. Significantly more microvessels in the subchondral bone of the femoral head were counted in the MP+Exo group compared with the MP group (Fig. 3D).

\section{Angiogenic enhancement by hiPS-MSC-Exos in HUVECs}

The in vitro results showed that hiPS-MSC-Exos could be incorporated into HUVECs, and that the incorporated hiPS-MSC-Exos could promote the proliferation, migration and tube formation of HUVECs.

\subsection{Internalization of hiPS-MSC-Exos into HUVECs}

Fluorescent images revealed that DiO-labeled hiPS-MSC-Exos (green) were located in the perinuclear region of HUVECs (Fig. 4A). This suggested that hiPS-MSC-Exos could be internalized by HUVECs. The phagocytosis phenomenon provides biological basis for the potential functional influence of hiPS-MSC-Exos on HUVECs.

\section{2. hiPS-MSC-Exos promote proliferation, migration} and tube formation of HUVECs in vitro

The proliferative capacity of HUVECs was significantly increased in the presence of hiPS-MSC-Exos at all time-points, and the effect was dose dependent (Fig. 4B). Migration capacity of
HUVECs was also enhanced 12 hours after stimulation by hiPS-MSC-Exos, and migration area was significantly increased in the Exo group compared with the control group (Fig. 4C and D). Moreover, hiPS-MSC-Exos markedly promoted tube formation of HUVECs at both 4 and 8 hours in the Exo group compared to the control group, and again the effect was dose dependent (Fig. 4E). The values of tubule lengths, branch points, covered area and total loops were all significantly higher in the Exo group compared with the control group (Fig. 4F).

\section{Activation of PI3K/Akt is involved in the promotion of angiogenesis by hiPS-MSC-Exos in vitro}

The PI3K/Akt signaling pathway of HUVECs was activated in response to iPS-MSC-Exos treatment. Inhibition of PI3K/Akt impaired the angiogenic effect of iPS-MSC-Exos on HUVECs.

\section{1. hiPS-MSC-Exos activate PI3K/Akt in HUVECs}

Akt, phosphorylated Akt and downstream genes and proteins of the PI3K/Akt signaling pathway were evaluated by qRT-PCR and western blotting. The results showed that the levels of BCL2A1, Sphk1, MYC and THBS1 were increased, whereas the expression of PTEN, RBL2 and CDKN1A were decreased in the Exo group compared with the control group (Fig. 5A and B). This suggested that hiPS-MSC-Exos significantly influenced PI3K/Akt signaling in HUVECs. Moreover, the result of western blotting identified an increased expression level of phosphorylated Akt protein, suggesting that 
hiPS-MSC-Exos activated PI3K/Akt signaling in HUVECs.

4.2. The effect of hiPS-MSC-Exos can be abolished by blockade of PI3K with LY294002

The PI3K/Akt signaling pathway was blocked in HUVECs by a PI3K inhibitor (LY294002; $10 \mu \mathrm{M}$ ), to determine whether exosomes still exerted their effect. The result of western blotting showed that
hiPS-MSC-Exos did not increase the expression of p-Akt protein following the application of LY294002. qRT-PCR and western blotting results revealed that the PI3K/Akt signaling-associated genes were down-regulated following the addition of LY294002 (Fig. 6A and B). This suggested that hiPS-MSC-Exos could not activate the PI3K/Akt signaling pathway after the pathway was blocked.
A

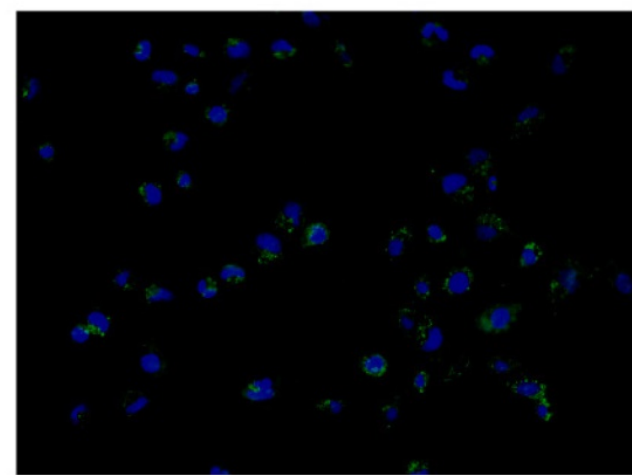

C

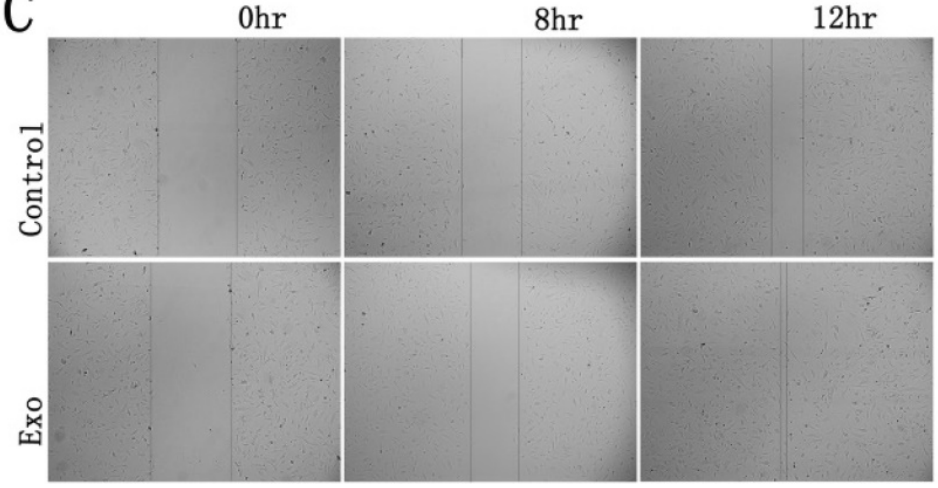

E

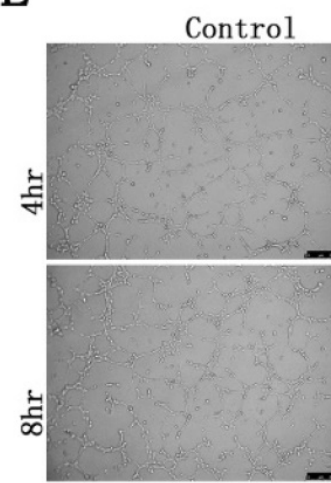

Ohr

$\operatorname{Exos}\left(1 \times 10^{10} / \mathrm{mL}\right)$

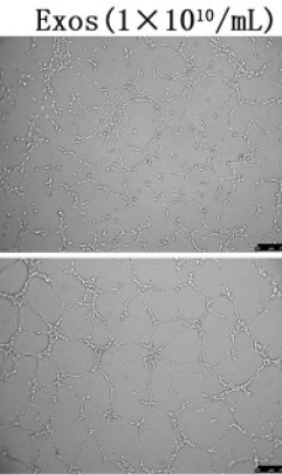

$\operatorname{Exos}\left(1 \times 10^{11} / \mathrm{mL}\right)$

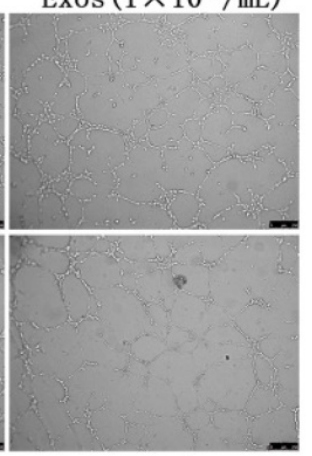

B

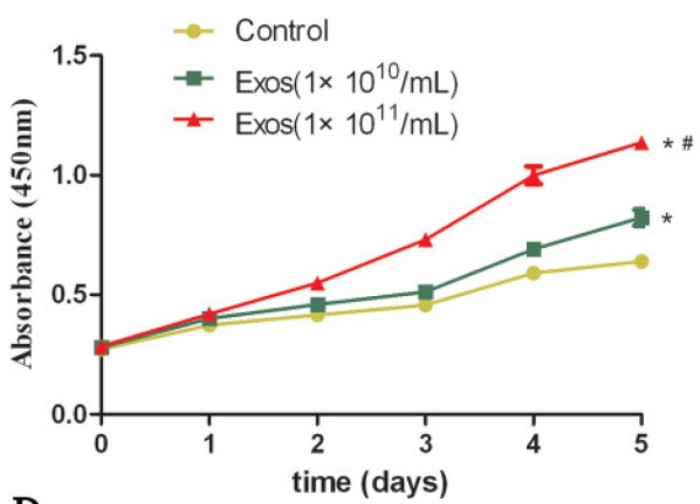

D

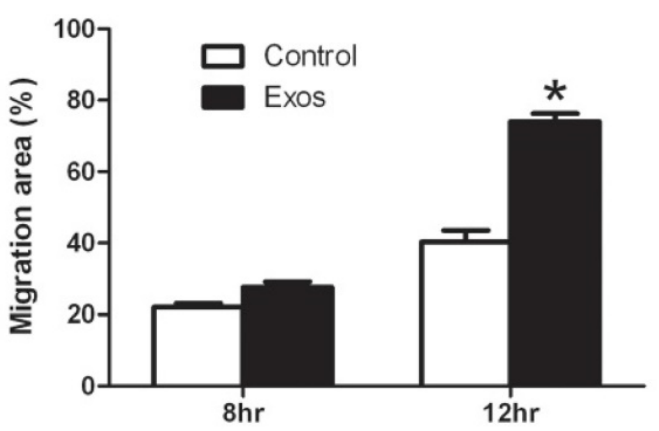

F
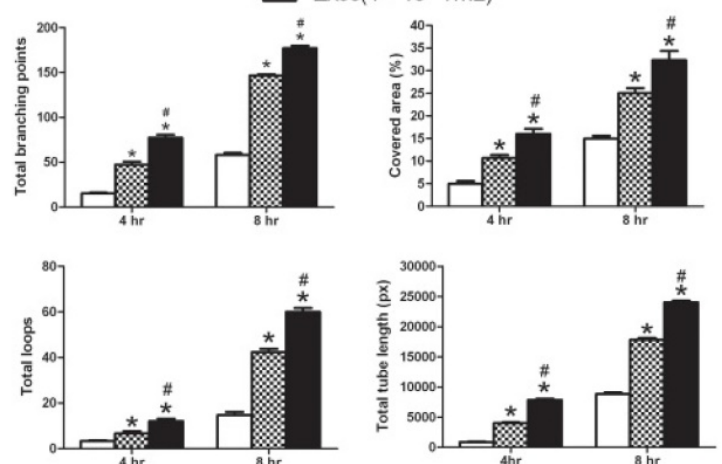

Figure 4. Functional influence of hiPS-MSC-Exos on HUVECs. (A) Fluorescent microscopy analysis. DiO-labeled hiPS-MSC-Exos (green) were located in the perinuclear region of HUVECs. (B) CCK-8 assay. hiPS-MSC-Exos significantly increased the proliferative ability of HUVECs in a dose-dependent manner. (C) Scratched wound assay. hiPS-MSC-Exos significantly enhanced the migration ability of HUVECs. (D) Quantitative analysis of the migration rate of HUVECs. The value of migration area was significantly greater in the Exo group than in the control group at $12 \mathrm{~h}$. (E) Capillary network formation assay. hiPS-MSC-Exos significantly enhanced tube formation ability in the Exo group compared to the control group in a dose-dependent manner. (F) Quantitative analysis of capillary network formation assay. The values of total branching points, total tube length, cell covered area, and total loops were all significantly higher in the Exo group than in the control group at 4 time point of 4 and 8 h. $*$ represents a statistically-significant difference compared with the control group $(P<0.05)$. \# represents statistically-significant difference compared with the Exos group $(1 \times 1010 / \mathrm{mL})(P<$ 0.05). 
A

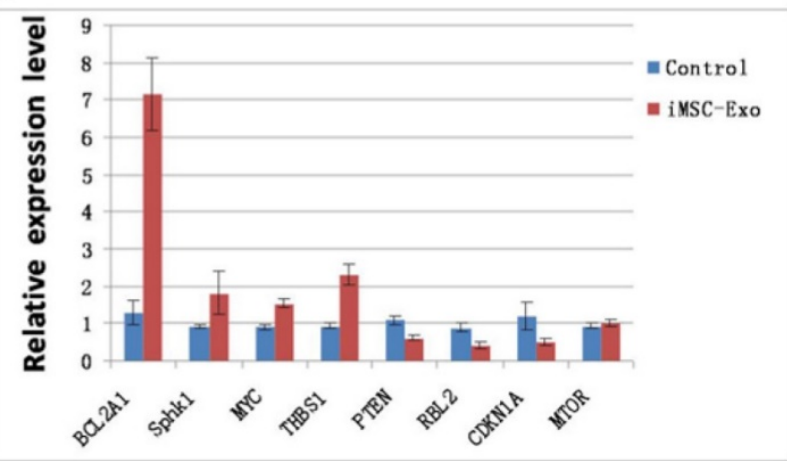

B

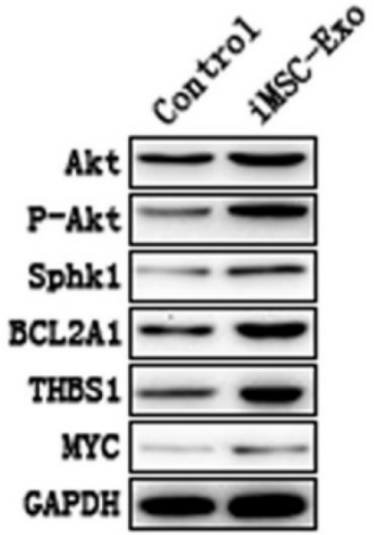

Figure 5. hiPS-MSC-Exos activate PI3K/Akt in HUVECs. (A) qRT-PCR analysis. Expression levels of PI3K/Akt signaling-related genes were significantly increased under the treatment of hiPS-MSC-Exos. (B) Western blot analysis. Phosphorylation of Akt protein and expression levels of PI3K/Akt signaling-related proteins were increased following treatment with hiPS-MSC-Exos.

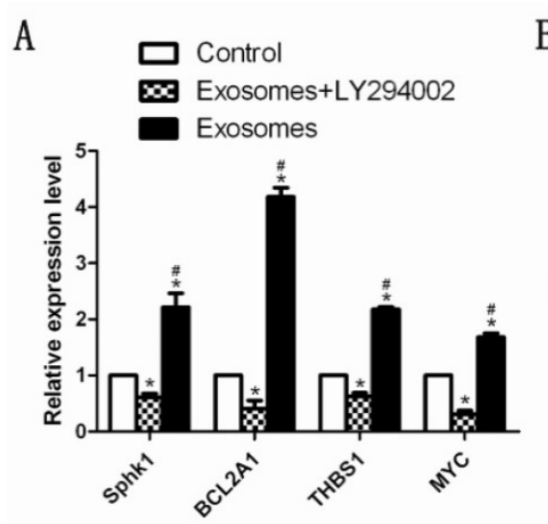

D

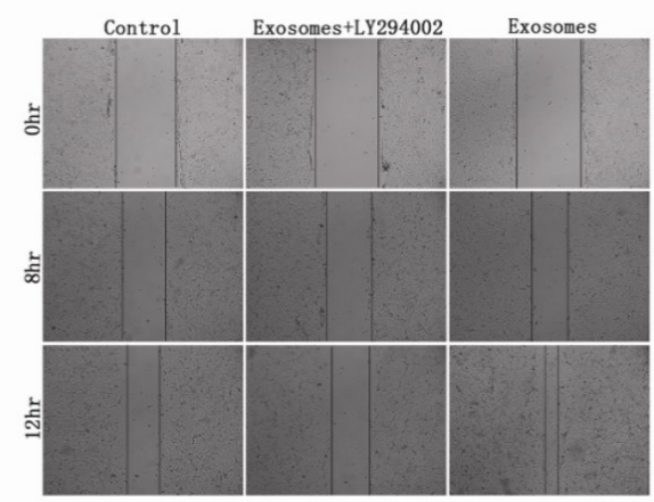

F

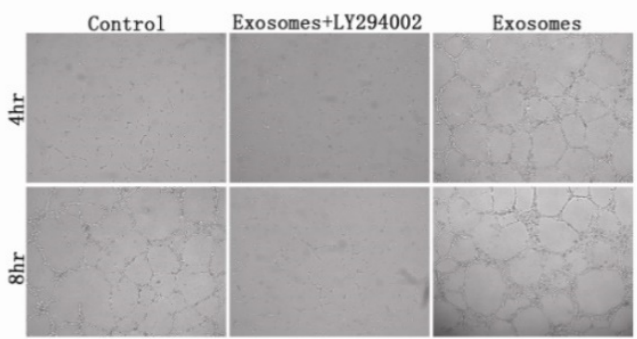

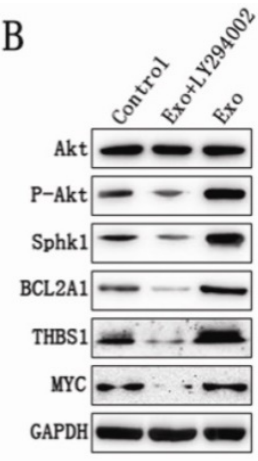

C

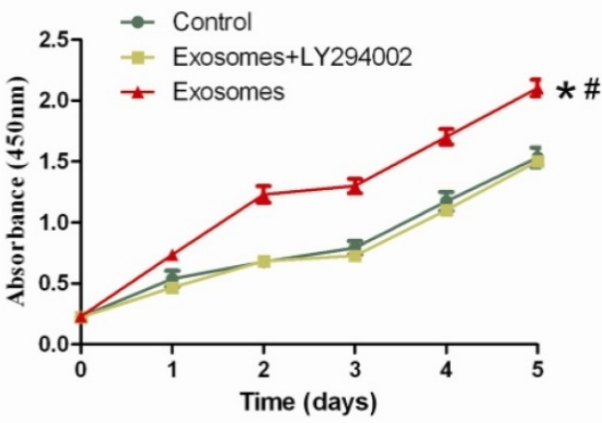

E

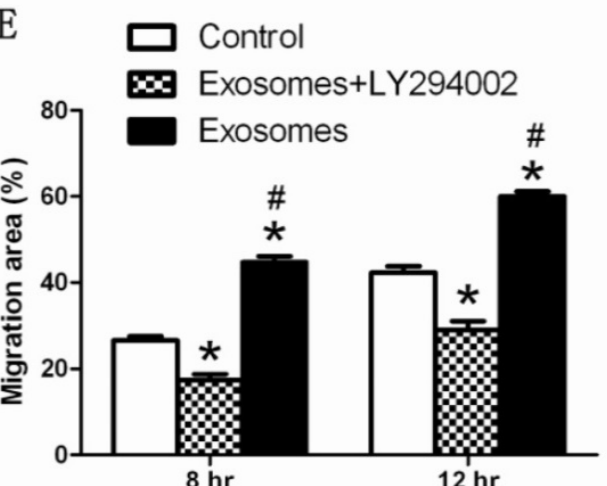

G

$\square$ Control

$\infty$ Exosomes+LY294002

Exosomes
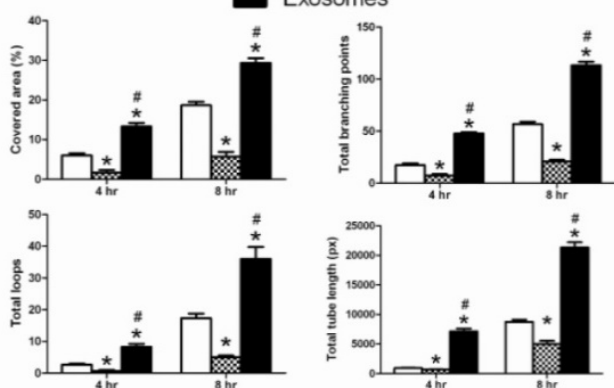

Figure 6. The effect of hiPS-MSC-Exos can be abolished by blockade of PI3K with LY294002. (A)(B) qRT-PCR and western blot analysis. hiPSC-MSC-Exos could not increase the expression levels of PI3K/Akt signaling-related molecules after blockade of PI3K with LY294002. (C) CCK-8 assay. The enhanced prolifierative ability conferred by hiPS-MSC-Exos was abolished by blockade of PI3K with LY294002. (D)(E) Scratched wound assay and quantitative analysis. The enhanced migration ability conferred by hiPS-MSC-Exos was abolished by blockade of PI3K with LY294002. (F)(G) Capillary network formation assay and quantitative analysis. The increased tube formation ability conferred by hiPS-MSC-Exos was abolished by blockade of PI3K with LY294002. * represents statistically-significant difference compared with the control group $(P<0.05)$. \# represents statistically-significant difference compared with the Exos+LY294002 group $(P<0.05)$. 
We next evaluated whether hiPS-MSC-Exos could still promote proliferation, migration and tube formation following the application of LY294002 on HUVECs. The result revealed that the proliferation, migration and tube formation capacities were significantly lower in the Exo+LY294002 group than in the Exo group and the control group (Fig 6C-6G). This suggested that hiPS-MSC-Exos might exert their angiogenic effect through activation of the PI3K/Akt signaling pathway in HUVECs.

\section{Discussion}

Local ischemia and secondary osteonecrosis are considered the core pathological mechanisms in osteonecrosis of the femoral head. There is still no effective treatment to repair the blood supply to the femoral head. Our study suggests for the first time that hiPS-MSC-Exos could promote local angiogenesis and prevent bone loss in a rat ONFH model. The in vitro results suggested that hiPS-MSC-Exos might increase the angiogenic effects of vascular endothelial cells through activation of the PI3K/Akt signaling pathway.

Stem cell transplantation has been proven to be able to promote angiogenesis in ONFH [7, 8]. However, the potential risks and problems are a major limitation for the possibility of clinical application. Exosomes secreted by stem cells have been demonstrated to show similar therapeutic effects on ischemic diseases. Arslan et al[34] reported that mesenchymal stem cell-derived exosomes could activate the PI3K/Akt pathway to enhance myocardial viability and prevent adverse remodeling after myocardial ischemia/reperfusion injury. Xin et al[35] found that systemic administration of exosomes released from mesenchymal stromal cells could promote functional recovery and neurovascular plasticity after stroke in rats. Besides the promising therapeutic effects, the new alternative method avoids all the risks and problems elicited by direct stem cell transplantation. Our previous studies identified the angiogenic effect of exosomes derived from hiPS-MSCs on ischemic diseases in limb ischemia and skin defects animal models [17, 18]. In this study, hiPS-MSC-Exos significantly inhibited the progression of osteonecrosis in a rat ONFH model. hiPS-MSC-Exos successfully stopped the destruction of trabecular bone, and increased local angiogenesis in the femoral head.

iPSCs are recognized as a promising cell source for their indefinite proliferation and multiple differentiation capacities similar to those of Embryonic stem cells (ESCs) [36]. Despite promising treatment potential of iPSCs, Kawamura's report identified the immunogenicity of this cell type [37].
Alternatively, previous studies have validated using MSCs as the immunomodulator for inflammation and autoimmunity, the major point of the application is low immunogenicity [38]. Thus in our study, we induced iPSCs into MSCs for the generation of exosomes. Our previous studies have identified that transplantion of exosomes derived from iPS-MSCs did not induce immune reactions in vivo $[17,18]$. Besides low immunogenicity, MSCs derived from iPSCs could generate even greater regenerative potential, which is attributed to superior survival and engraftment after transplantation [39]. Moreover, iPS-MSCs do not express pluripotency genes such as Nanog and Oct4, which are believed to be essential for the functional maintenance of iPSCs [40]. The extraction of exosomes was performed by a combination of ultrafiltration and purification according to our previous method [18]. The obtained exosomes exhibited round-shaped morphology with size ranging from 50 to $150 \mathrm{~nm}$, and expressed typical exosomal surface markers including CD9, CD63 and CD81. The concentration of extracted exosomes reached $3.4 \times 10^{12} / \mathrm{mL}$, which were generated from 1 $\times 10^{7}$ cells. Our studies applied two concentrations for the in vivo and in vitro experiments: $1 \times 10^{10} / \mathrm{mL}$ and 1 $\times 1011 / \mathrm{mL}$. The results suggested that both concentrations showed therapeutic effects on ONFH, and that the effects of exosomes were dose dependent.

The potential angiogenic mechanism of hiPS-MSC-Exos was also investigated in this study. The most important function of exosomes is their role in communication from host cells to target cells. The contents of exosomes are composed of functional biochemicals including mRNA, microRNAs, and proteins. We found that hiPS-MSC-Exos could access endothelial cells to enhance their proliferation, migration and tube-forming capacities. The ability of endothelial cells to migrate and form capillary-like structures is essential for angiogenesis in vivo [41]. We assumed that hiPS-MSC-Exos might activate a specific molecular pathway of endothelial cells to exert angiogenic effects. It is well known that the PI3K/Akt signaling pathway plays a central role in stem cell-regulated cell survival, proliferation, migration and angiogenesis [5]. Activation of the PI3K/Akt signaling pathway can significantly enhance the functions of endothelial cells in various tissues [36, 42]. In this study we found that hiPS-MSC-Exos could activate the PI3K/Akt signaling pathway in endothelial cells. However when the pathway was blocked by a PI3K inhibitor, the beneficial effect elicited by hiPS-MSC-Exos was soon abolished. These results suggest that hiPS-MSC-Exos might increase the angiogenic ability of endothelial cells through activation of the PI3K/Akt signaling pathway. 


\section{Conclusions}

We identified for the first time that hiPS-MSCs-Exos prevent bone loss and promote angiogenesis of the femoral head in ONFH. The effects might be due to that hiPS-MSC-Exos activate the PI3K/Akt signaling pathway of vascular endothelial cells to enhance proliferation, migration and tube-forming capacities. Our findings suggest that hiPS-MSCs-Exos may be a promising therapeutic approach in the treatment of ONFH.

\section{Abbreviations}

ONFH, osteonecrosis of the femoral head; iPSCs, induced pluripotent stem cells; iPS-MSC-Exos, induced pluripotent stem cell-derived mesenchymal stem cells; MP, methylprednisolone acetate; BV/TV, bone volume/total volume; Tb.Th, trabecular thickness; Tb.N, trabecular number; HUVECs, human umbilical vascular endothelial cells.

\section{Acknowledgement}

This research was supported by the National Nature Science Foundation of China (Grant nos. 81472152, 81371962, and 81171861), and Joint Project Funding for Major Diseases in Shanghai (Grant nos. 2014ZYJB0301).

\section{Authors' Contributions}

Yang Wang and Qing Li were in charge of the research design, as well as data interpretation and critical revising of the paper. Xiaolin Liu, Qing Li, Xin $\mathrm{Niu}$ and $\mathrm{Bin} \mathrm{Hu}$ were involved in the in vitro experiments, as well as data acquisition, processing and interpretation. Xiaolin Liu, Wenqi Song and Jian Ding and Shengbao Chen were involved in the in vivo studies, as well as obeservation and evaluation of the treating effects. Xiaolin Liu drafted the paper. Yang Wang and Changqing Zhang gave approval of the submitted and final versions. All the authors have read and approved the final submitted manuscript.

\section{Conflicts of Interest}

Xiaolin Liu, Qing Li, Xin Niu, Bin Hu, Shengbao Chen, Wenqi Song, Jian Ding, Changqing Zhang and Yang Wang declare that they have no conflict of interest.

\section{References}

1. Katsuda T, Kosaka N, Takeshita F. The therapeutic potential of mesenchymal stem cell-derived extracellular vesicles. Proteomics. 2013 May;13(10-11):1637-53

2. Mont MA, Hungerford DS. Non-traumatic avascular necrosis of the femoral head. J Bone Joint Surg Am. 1995;77:459-474.

3. Feng Y, Yang SH, Xiao BJ, et al. Decreased in the number and function of circulation endothelial progenitor cells in patients with avascular necrosis of the femoral head. Bone. 2010 Jan;46(1):32-40.

4. Mankin HJ. Nontraumatic necrosis of bone (osteonecrosis). N Engl J Med. 1992;326:1473-9.
5. Chen J, Crawford $\mathrm{R}$, Chen $\mathrm{C}$, et al. The key regulatory roles of the PI3K/Akt signaling pathway in the functionalities of mesenchymal stem cells and applications in tissue regeneration. Tissue Eng Part B Rev. 2013 Dec;19(6):516-28.

6. Grunewald M, Avraham I, Dor Y, Bachar-Lustig E, Itin A, Jung S, et al. VEGF-induced adult neovascularization: recruitment, retention, and role of accessory cells. Cell. 2006;124:175

7. Yan Z, Hang D, Guo C, Chen Z. Fate of mesenchymal stem cells transplanted to osteonecrosis of femoral head. J Orthop Res. 2009 Apr;27(4):442-6.

8. Tabatabaee RM, Saberi S, Parvizi J, Mortazavi SM, Farzan M. Combining Concentrated Autologous Bone Marrow Stem Cells Injection With Core Depression Improves Outcome for Patients with Early-Stage Osteonecrosis of the Femoral Head: A Comparative Study. J Arthroplasty. 2015 Sep;30(9 Suppl):11-5.

9. Balsam LB, Wagers AJ, Christensen JL, et al. Haematopoietic stem cells adopt mature haematopoietic fates in ischaemic myocardium. Nature. $2004 \mathrm{Apr}$ 8;428(6983):668-73

10. Ratajczak MZ, Jadczyk T, Pedziwiatr D, Wojakowski W. New advances in stem cell research: practical implications for regenerative medicine. Pol Arch Med Wewn. 2014;124(7-8):417-26.

11. Azmi AS, Bao B, Sarkar FH. Exosomes in cancer development, metastasis, and drug resistance: a comprehensive review. Cancer Metastasis Rev. 2013; 32(3-4):623-42

12. Properzi F, Ferroni E, Poleggi A, et al. The regulation of exosome function in the CNS: implications for neurodegeneration. Swiss Med Wkly. 2015; 12(145):w14204

13. Rosca AM, Rayia DM, Tutuianu R. Emerging Role of Stem Cells Derived Exosomes as Valuable Tools for Cardiovascular Therapy. Curr Stem Cell Res Ther. 2015 Oct 26.

14. Takeshi Katsuda, Nobuyoshi Kosaka, Fumitaka Takeshita, et al. The therapeutic potential of mesenchymal stem cell-derived extracellular vesicles. Proteomics. 2013, 13:1637-1653.

15. Chaput N, Thery C. Exosomes: Immune properties and potential clinical implementations. Semin Immunopathol. 2011 Sep;33(5):419-40.

16. Breakefield XO, Frederickson RM, Simpson RJ. Gesicles: Microvesicle "cookies" for transient information transfer between cells. Mol Ther. 2011 Sep;19(9):1574-6.

17. Zhang J, Guan J, Niu X, et al. Exosomes released from human induced pluripotent stem cells-derived MSCs facilitate cutaneous wound healing by promoting collagen synthesis and angiogenesis. J Transl Med. 2015; 13: 49.

18. Hu GW, Li Q, Niu X, et al. Exosomes secreted by human-induced pluripotent stem cell-derived mesenchymal stem cells attenuate limb ischemia by promoting angiogenesis in mice. Stem Cell Res Ther. 2015 Apr 10;6:10.

19. Piao $W$, Wang $H$, Inoue $M$, Hasegawa $M$, Hamada $H$, and Huang J. Transplantation of Sendai viral angiopoietin-1-modified mesenchymal stem cells for ischemic limb disease. Angiogenesis. 2010;13:203.

20. Hung SC, Pochampally RR, Chen SC, Hsu SC, and Prockop DJ. Angiogenic effects of human multipotent stromal cell conditioned medium activate the PI3K-Akt pathway in hypoxic endothelial cells to inhibit apoptosis, increase survival, and stimulate angiogenesis. Stem Cells. 2007;25:2363.

21. Liao J, Wu Z, Wang Y, Cheng L, Cui C, Gao Y, et al. Enhanced efficiency of generating induced pluripotent stem (iPS) cells from human somatic cells by a combination of six transcription factors. Cell Res. 2008;18:600-3.

22. Cai J, Li W, Su H, Qin D, Yang J, Zhu F, et al. Generation of human induced pluripotent stem cells from umbilical cord matrix and amniotic membrane mesenchymal cells. J Biol Chem. 2010;285:11227-34.

23. Rowland TJ, Miller LM, Blaschke AJ, et al. Roles of integrins in human induced pluripotent stem cellll grrowth on Matrigel and vitronectin. Stem Cells and Development. 2010; 19:1231-1240.

24. Zou L, Luo $\mathrm{Y}$, Chen $\mathrm{M}$, et al. A simple method for deriving functional MSCs and applied for osteogenesis in 3D scaffolds. Scientific Reports. 2013; 3:2243.

25. Kosaka N, Yoshioka Y, Hagiwara K, Tominaga N, Ochiya T. Functional analysis of exosomal microRNA in cell-cell communication research. Methods Mol Biol. 2013, 1024:1-10.

26. Montecalvo A, Larregina AT, Morelli AE. Methods of analysis of dendritic cell-derived exosome-shuttle microRNA and its horizontal propagation between dendritic cells. Methods Mol Biol. 2013:1024:19-40.

27. Properzi F, Logozzi M, Fais S. Exosomes: the future of biomarkers in medicine. Biomarkers in Medicine. 2013, 7:769-778.

28. Bitto A, Polito F, Burnett B, et al. Protective effect of genistein aglycone on the development of osteonecrosis of the femoral head and secondary osteoporosis induced by methylprednisolone in rats. J Endocrinol. 2009;201:321-328.

29. Han N, Yan Z, Guo CA, et al. Effects of p-glycoprotein on steroid-induced osteonecrosis of the femoral head. Calcif Tissue Int. 2010;87:246-253.

30. Sun Y, Feng Y, Zhang C, Cheng X, Chen S, Ai Z, Zeng B: Beneficial effect of autologous transplantation of endothelial progenitor cells on steroid-induced femoral head osteonecrosis in rabbits. Cell Transplant. 2011;20:233-243.

31. Sun Y, Feng Y, Zhang C: The effect of bone marrow mononuclear cells on vascularization and bone regeneration in steroid-induced osteonecrosis of the femoral head. Joint Bone Spine. 2009;76:685-690.

32. Weidner N, Semple JP, Welch WR, et al. Tumor angiogenesis and metastasis-correlation in invasive breast carcinoma. $N$ Engl J Med. 1991;324:1-8.

33. [33] Pang H, Flinn R, Patsialou A, Wyckoff J, Roussos ET, Wu H, Pozzuto M, Goswami S, Condeelis JS, Bresnick AR: Differential enhancement of breast cancer cell motility and metastasis by helical and kinase domain mutations of class IA phosphoinositide 3-kinase. Cancer Res. 2009;69:8868-8876. 
34. [34] Arslan F, Lai RC, Smeets MB, Akeroyd L, Choo A, Aguor EN, et al. Mesenchymal stem cell-derived exosomes increase ATP levels, decrease oxidative stress and activate PI3K/Akt pathway to enhance myocardial viability and prevent adverse remodeling after myocardial ischemia/reperfusion injury. Stem Cell Res. 2013;10:301-12.

35. Xin H, Li Y, Cui Y, Yang JJ, Zhang ZG, Chopp M. Systemic administration of exosomes released from mesenchymal stromal cells promote functional recovery and neurovascular plasticity after stroke in rats. J Cereb Blood Flow Metab. 2013;33:1711-5

36. Takahashi K, Yamanaka S. Induction of pluripotent stem cells from mouse embryonic and adult fibroblast cultures by defined factors. Cell. 2006;126(4):663-76.

37. Kawamura T, Miyagawa S, Fukushima S, et al. Cardiomyocytes Derived from MHC-Homozygous Induced Pluripotent Stem Cells Exhibit Reduced Allogeneic Immunogenicity in MHC-Matched Non-human Primates. Stem Cell Reports. 2016 Mar 8;6(3):312-20.

38. Zhang $X$, Jiao C, Zhao S. Role of mesenchymal stem cells in immunological rejection of organ transplantation. Stem Cell Rev. 2009;4:402-409.

39. Lian $\mathrm{Q}$, Zhang $\mathrm{Y}$, Zhang J, Zhang $\mathrm{HK}, \mathrm{Wu} \mathrm{X}$, Zhang $\mathrm{Y}$, et al. Functional mesenchymal stem cells derived from human induced pluripotent stem cells attenuate limb ischemia in mice. Circulation. 2010;121:1113-23.

40. Yu J, Vodyanik MA, Smuga-Otto K, Antosiewicz-Bourget J, Frane JL, Tian S, et al. Induced pluripotent stem cell lines derived from human somatic cells. Science. 2007;318:1917-20.

41. Carmeliet P. Mechanisms of angiogenesis and arteriogenesis. Nat Med. 2000;6:389-395.

42. Graupera M, Guillermet-Guibert J, Foukas LC, et al. Angiogenesis selectively requires the p110alpha isoform of PI3K to control endothelial cell migration. Nature. 2008;453:662-666. 